\title{
A New Approach for Modeling Piezoresistive Force Sensors Based on Semi-Conductive Polymer Composites
}

\author{
Masoud Kalantari, Javad Dargahi, Jozsef Kövecses, Mahmood Ghanbari, and Shahrzad Nouri
}

\begin{abstract}
Semi-conductive polymer composites are used in a wide range of sensors, measurement devices. This paper discusses the development of a model and a new theoretical formulation for predicting piezoresistive behavior in semi-conductive polymer composites including their creep behavior and contact resistance. The relationship between electrical resistance and force applied to the piezoresistive force sensor can be predicted by using the proposed theoretical formulation. In order to verify the proposed formulation, the piezoresistive behavior of Linqstat, a carbonfilled polyethylene, was modelled mathematically. In addition, some experimental tests such as Thermo Gravitational Analysis and Scanning Electron Microscopy have been performed on Linqstat to find the volume fraction and size of carbon particles which are essential for modeling. In addition, on a fabricated force sensor using Linqstat, a the force vs. resistance curve was obtained experimentally which verified the validity and reliability of the proposed formulation.
\end{abstract}

Index Terms-Semiconductor device modeling, Semiconductor materials measurements, Piezoresistive devices, Biomedical transducers.

\section{INTRODUCTION}

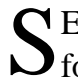
EMI conductive polymer composites are widely used as force and pressure sensors [1-7]. Most recently, Vanello et al. proposed a biomimetic-fabric-based sensing glove which is used for monitoring hand posture and gesture. They used a network of piezoresistive force sensors based on semi-

This research was supported by the Natural Sciences and Engineering Research Council of Canada (NSERC). The support is gratefully acknowledged.

M. Kalantari is with the Department of Mechanical and Industrial Engineering of Concordia University and Centre for Intelligent Machines, McGill University, Montreal, Quebec H3G 2W1 Canada, (e-mail: m_kalan@encs.concordia.ca, phone 514-848 2424).

J. Dargahi is with the Department of Mechanical and Industrial Engineering, Concordia University, Montreal, Quebec H3G 2W1 Canada, (email:dargahi@encs.concordia.ca)

J. Kövecses is with the Department of Mechanical Engineering and Centre for Intelligent Machines, McGill University, Montreal, Quebec H3A 2K6 Canada, (e-mail: jozsef.kovecses@mcgill.ca)

M. Ghanbari is with the Department of Mechanical and Industrial Engineering, Concordia University, Montreal, Quebec H3G 2W1 Canada, (email: m_ghanba@encs.concordia.ca

S. Nouri is with the Department of Mechanical and Industrial Engineering, Concordia University, Montreal, Quebec H3G 2W1 Canada, (e-mail: sh_nouri@encs.concordia.ca conductive polymer composites [8]. In another research work, Kawasaki et al. used distributed tactile sensors with 624 detecting points as the sensing element of a robot hand, named Gifu II [9]. Their distributed tactile sensor consists of a matrix of piezoresistive force sensors with semi-conductive polymer composite elements. At the National Taiwan University another similar robotic hand (the NTU hand), was developed by Lin et al. who used the same type of sensors for robotic tactile perception [10].

Semi-conductive polymer composites are suitable for use as pressure distribution sensors because of their simple structure in different applications [11-12]. Semi-conductive polymer composites exhibit change in electrical resistivity caused by change in the force applied to their structure. Semi-conductive polymer composites consist of impregnated nano-scale conductor particles inside the structure of a nonconductive material. The micro structure of conductor-filled polymer composites can be classified among the random whisker composites [13]. This is due to the fact that the conductive particles or fillers are randomly dispersed inside the structure of a non-conductive matrix. The change of electrical resistance in semi-conductive polymer composites occurs when changing the distance between conductor particles inside the matrix [14]. Wang et al. proposed a mathematical model for piezoresistivity of carbon-black-filled silicone rubber based on differences in carbon black contents [15]. They proposed a model of a piezoresistivity curve which varies with different carbon black contents based on a shell model and tunneling current. Xie et al. [16] proposed a model for carbon black filled polymers under elongations. Hall et al. [17] proposed a calibration method for eliminating drift in Force Sensing Resistors (FSR). In their proposed method, the drift of a force sensor was compensated by signal conditioning. However, they did not provide the theoretical basis for the drift for FSRs. Xiang et al. [18] proposed a model for predicting time dependency and piezoresistivity of conductor filled polymer composites using inter-particle separation change under applied pressure. Their developed piezoresistance model, however does not take into account contact resistance. In addition, their proposed method for modeling creep is not suitable for polymer composites displaying viscoelastic behavior.

This paper presents an improved and more comprehensive 
mathematical model between force applied to semi-conductive polymer composites and the consequent change of electrical resistance. In addition, the contact resistance between the electrodes and polymer composites is considered in the formulation. Semi-conductive polymer composite-made force sensors show drift in their output. The reason for this drift in such force sensors could be due to the creep behavior of the semi-conductive polymer composite used as the sensing element. If a polymer composite such as Linqstat, (a patented name), is subjected to a constant force, it exhibits creep behavior which causes the drift in the output of the force sensor [18]. In order to include the drift of the force sensor in the formulation, a viscoelastic model is used to represent semiconductive polymer composites based on their creep behavior. Hence, the strain rate of semi-conductive polymer composites is formulated and used in the proposed formulation.

To verify the validity of the proposed formulation, an experimental setup was prepared and various tests were performed. Linqstat, as an industrial semi-conductive polymer composite, was selected to be tested. Furthermore, an accurate force sensor made from Linqstat material was developed and calibrated. The curve of force versus resistance for the sensor was determined and compared with the proposed formulation. In addition, a creep test was performed on Linqstat and the results were compared to the model predictions. Finally, it is shown that the proposed formulation is accurate and reliable for predicting the output of these piezoresistive force sensors fabricated from polymer composites used as sensing elements.

\section{DEVELOPMENT OF THE PROPOSED FORMULATION}

\section{A. Review of related formulation}

In semi-conductive polymer composites, the matrix is selected from non-conductive polymers, while the filler is selected from conductive materials. By subjecting the polymer composite to compressive stress, the filler particles inside the matrix start to display micro-Brownian motion [15]. The fillers move closer to each other because of the strain in the matrix that causes a change in the electrical resistance the polymer composite. There are two main types of resistances that cause a change in resistivity within the composite, namely constriction resistance and tunneling resistance. It has been shown [14] that the total resistance of the polymer composites can be given as

$$
R=\frac{(L-1) R_{m}+L R_{c}}{S}
$$

where $R_{m}$ is the resistance between two adjacent filler particles, $R_{c}$ is the resistance across a single filler particle, $L$ is the number of particles forming one conductive path, and $S$ is the total number of effective conduction paths. The tunneling current would flow through a gap between adjacent particles [19]. The tunneling current at low applied voltages [20] can be written as:

$$
J=\frac{3 \sqrt{2 m \varphi}}{2 s}\left(\frac{q}{h}\right)^{2} V e^{-\left(\frac{4 \pi \mathrm{s}}{\mathrm{h}} \sqrt{2 m \varphi}\right)}
$$

where $q$ and $m$ are electron charge and mass respectively, $\varphi$ is the height of the potential barrier between two adjacent filler particles, $h$ is Plank's constant, $V$ is the applied voltage, and $s$ is the thickness of the insulating film. The height of the potential barrier can be extracted from the work function of the polymer. The $\varphi$ between adjacent particles in a composite can be calculated by subtraction of the polymer (matrix) work function and the filler work function. In this current project, carbon black and polyethylene is used as the filler and matrix respectively. Therefore, the required potential height of carbon black and polyethylene is considered as $0.05(\mathrm{eV})$ [21]. The tunneling resistivity is proportional to area $a^{2}[18]$ of the contact between two filler particles,

$$
R_{m}=\frac{\rho_{m}}{a^{2}}=\frac{V}{a^{2} J}=\frac{8 \pi s}{3 a^{2} \gamma q^{2}} e^{\gamma s}
$$

where $J$ is the tunneling current, and

$$
\gamma=\frac{4 \pi}{h} \sqrt{2 m \varphi}
$$

By putting $R_{m}$ in (1), and equating $L-1 \sim L,[18]$ the total resistance can be introduced as

$$
R=\frac{L}{S}\left[\frac{8 \pi s}{3 a^{2} \gamma q^{2}} e^{\gamma s}\right]+\frac{L}{S} R_{c}
$$

In this current research work, carbon black is selected to be the filler particle inside the matrix. The matrix is selected to be a nonconductive material with high resistance. Therefore, the resistance of carbon black, $R_{c}$, which is highly conductive can be considered negligible. So, $L / S R_{c}$ in the above equation is omitted for the coming derivations. By dividing the actual resistance with the initial resistance [18], the following formula can be obtained.

$$
\frac{R}{R_{0}}=\frac{s}{s_{0}} e^{-\gamma\left(s_{0}-s\right)}
$$

where $R_{0}$ is the initial resistance of the composite, and $s_{0}$ is the initial distance between two adjacent filler particles. As a result of application of stress to the composite, it is also assumed that only the polymer (matrix) would carry the load. The deformation of the filler particles is in the range of a few nanometers, which is negligible in comparison with that of the matrix. The inter-particle separation distance [18] can be simply shown as

$$
s=s_{0}(1-\varepsilon)
$$


where $\varepsilon$ is the strain of the polymer matrix which could be considered time dependent. Wu [22] showed that the interparticle separation between two spherical fillers can be calculated from the following relation

$$
s_{0}=D\left[\left(\frac{\pi}{6 \phi}\right)^{1 / 3}-1\right]
$$

where $D$ is the filler particle diameter, and $\phi$ is the volume fraction of filler particles. By substituting (7) and (8) into (6), similar to that shown in [18], it can be written that

$$
\frac{R}{R_{0}}=(1-\varepsilon) e^{-\gamma D \varepsilon\left[\left(\frac{\pi}{6 \phi}\right)^{1 / 3}-1\right]}
$$

As mentioned earlier, $R_{0}$ is the initial resistance of the polymer composite which can be measured by precise measurement techniques.

\section{B. Working principle of a piezoresistive force sensor}

In a typical force sensor that uses polymer composites, a thin layer of the polymer composite is sandwiched between two layers of electrode. The electrodes are then covered with two layers of polyester films. Figure 1.a shows the schematic view of the components of such a sensor.
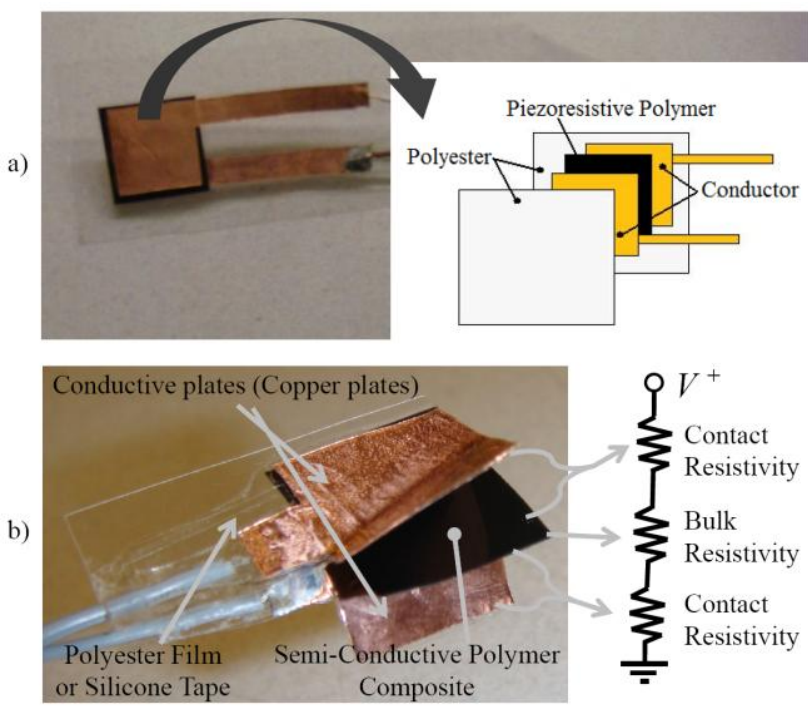

Fig. 1.a. View of the fabricated force sensor using Linqstat and its components $\mathrm{b}$. The schematic view of current flow geometry

When a compressive force is applied to the surface of the force sensor, its resistance drops due to a decrease in the resistance of the piezoresistive polymer, and also due to a slight decrease in the contact resistance between the conductors and the piezoresistive polymer. In other words, applying force causes a decrease in distance between filler particles inside the matrix, and an increase in the number of conductive paths which leads to a decrease in the resistance of polymer composites. Because there is no adhesive between the electrodes and the polymer in the fabrication process, when force is applied air between the electrode and polymer would leave from the air vent in the tail of the sensor. This results in a sudden decrease in contact

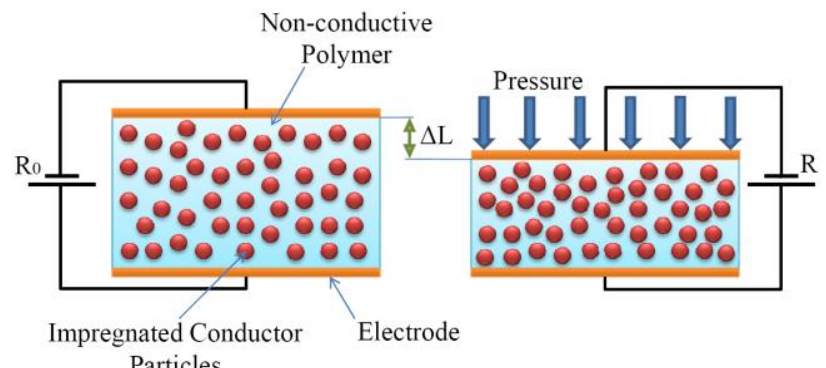

Fig. 2. The schematic view of the piezoresistive force sensor based on semi-conductive polymer composites materials. The figure shows the working principle of the sensor.

resistance between the electrodes and the polymer composite. As shown schematically in Fig. 2, the average distance between conductor particles would decrease by applying force to the sensor.

\section{Contact resistance}

In order to obtain an accurate reading of the force sensor output, the contact resistance between electrodes and the polymer composite was taken into account. It has been shown that the effective contact area between two adjacent members is only a small fraction of the apparent macroscopic area of contact [23]. Looking at the contact interface on a microscopic scale, the roughness of surfaces would be evident. Therefore, the electrical contact would take place on both separable mating surfaces through asperities, or a-spots [24]. In the present work, it is assumed that there is no film resistance caused by thin oxide layers on the contact surfaces since the polymer, polyethylene, does not oxide in the room temperature. Therefore, based on this assumption total resistance of the force sensor which is read by a measurement system attached to the sensor can be written as

$$
R_{\text {total }}=2 R_{\text {Con }}+R_{\text {Pol }}
$$

where $R_{\text {total }}$ is the total resistance of the sensor, $R_{\text {Con }}$ is the contact resistance between each electrode and polymer composite, and $R_{P o l}$ is the resistance of the polymer composite which is to be determined. Figure 1.b shows the schematic view of the total electrical resistance of the sensor.

By using the Holm and Greenwood formula [25], the contact resistance can be written as:

$$
R_{\text {Con }}=\frac{\rho_{1}+\rho_{2}}{4 n a}
$$

where $\rho_{1}$ and $\rho_{2}$ are the electrical resistivity of the two materials in contact, $a$ is the radius of a-spot, and $\mathrm{n}$ is the number of a-spots. The number and the radius of a-spots increase when force is applied to the contact surfaces. Figure 3 shows the change in the number of electrical contacts and the change of the interface of two adjacent members upon applying force. 

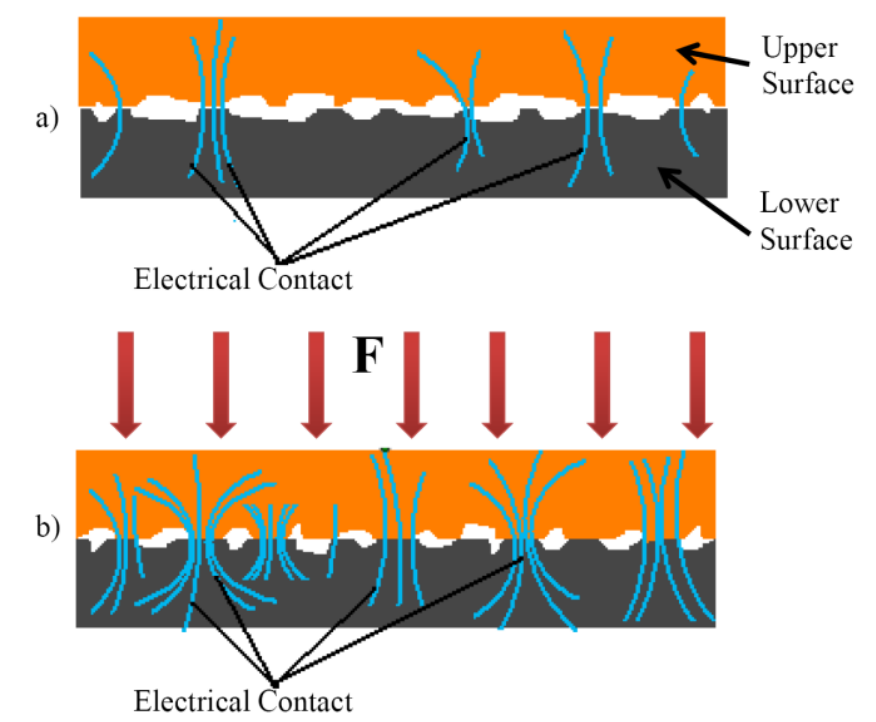

Fig.3. The schematic view of current path through contact a. unloaded contact area $\quad$ b. loaded contact area

All of the a-spots can be assumed as having an effective contact area which is affected by the applied force [26]. An increase in this applied force results in a larger effective area. From Fig. 3, it can be seen that when force is applied, there still exists some area between the two surfaces which are not in contact, known as ineffective regions. The effective area could be written as the summation of all a-spots.

Holm [23] presented a formula about contact resistance between different members with contact forces of 0 to $100 \mathrm{~N}$ as:

$$
R_{\text {Con }}=\frac{\rho_{1}+\rho_{2}}{4} \sqrt{\frac{\pi H}{F}}
$$

where $\rho_{1}$ and $\rho_{2}$ are the electrical resistivity of the two materials, $F$ is the applied force, and $H$ is the Meyer hardness of the softer member.

\section{Resistance of semi-conductive polymer composite by considering contact resistance}

The equivalent resistance of a piezoresistive sensor can be derived using the formulation presented in the previous sections. The following shows the total change in resistance of a semi-conductive polymer composite caused by applied load. By substituting (9) and (12) into (10), we can write

$$
\begin{aligned}
& R_{\text {total }}=2 R_{\text {Con }}+R_{\text {Pol }}= \\
& \frac{\rho_{1}+\rho_{2}}{2} \sqrt{\frac{\pi H}{F}}+R_{0}(1-\varepsilon) e^{-\gamma D \varepsilon\left[\left(\frac{\pi}{6 \phi}\right)^{1 / 3}-1\right]} \\
& R_{\text {Con }}=\frac{\rho_{1}+\rho_{2}}{4} \sqrt{\frac{\pi H}{F}} \quad, R_{P o l}=R_{0}(1-\varepsilon) e^{-\gamma D \varepsilon\left[\left(\frac{\pi}{6 \phi}\right)^{1 / 3}-1\right]} \\
& \text { and } \gamma=\frac{4 \pi}{h} \sqrt{2 m \varphi}
\end{aligned}
$$

where $\rho_{1}$ and $\rho_{2}$ are the electrical resistivity of two materials in contact, $H$ is the Meyer hardness of the softer member, $F$ is the applied force, $R_{0}$ is the initial resistance of the semiconductive polymer composite, $\varepsilon$ is the strain, $D$ is the filler particle diameter, $\phi$ is the volume fraction of the filler particles, $h$ is Plank's constant, $m$ is electron mass, and $\varphi$ is the height of the potential barrier between two adjacent filler particles. The measured parameters are listed in Table I.

\section{E. Modeling creep behavior}

After reviewing the total resistance results from (13), it is clear that the total resistance of the sensor is a function of the material properties of the semi-conductive polymer composite, the applied force, and the induced strain. The strain in (13) is considered constant for constant forces. However, in a real situation, the strain changes slightly with time while having a constant force on the material. In other words for a constant force, the strain is not constant due to the creep, and will change with time. The reason for this is that when a constant force is applied, semi-conductive polymer composites display creep behavior due to the viscoelastic properties inherent within all polymers. The creep of the polymer composites appears as drift in the force sensor output. Therefore, by applying a constant force to the force sensor, the resistivity decreases with time. For the prediction of creep behavior in semi-conductive polymer composites a model is developed based on spring-damper elements. Polymers behave as an elastic solid in some instances, and as a viscous fluid in other cases; they can generally be considered using viscoelastic material models [27]. A single Maxwell or a single KelvinVoigt element cannot represent the transient and instantaneous response of polymers accurately. Hence, using a combination of the two elements is recommended [28-31]. The strain in a creep test of semi-conductive polymer composites, such as Linqstat which is used when fabricating a force sensor, tends towards a constant value after a longer period of time. In fact, the impregnated carbon particles inside the polyethylene matrix, which is a thermoplastic polymer, change the mechanical properties of the composite similar to those of thermosets. These results were obtained after several accurate creep tests on Linqstat using a BOSE ELECTROFORCE 3200 device. Therefore, a standard linear solid model with threeparameters, shown in Fig. 4.a, is used for modeling the creep in semi-conductive polymer composites such as Linqstat. In fact the creep curves of both the Linqstat and standard linear solid model are very similar to each other, due to the fact that the strain in both of them tends toward a constant value with time. Since the creep behaviour of the Linqstat is being modeled, the standard linear solid model becomes the best fit for this purpose. Figure 4.a illustrates the selected lumpedparameter model and the creep curve for the standard linear solid model as well.

The constitutive equation of the standard linear solid model, governing the behaviour of the element, is written as [27] 


$$
\sigma+\frac{\mu_{1}}{E_{0}+E_{1}} \dot{\sigma}=\frac{E_{0} E_{1}}{E_{0}+E_{1}} \varepsilon+\frac{\mu_{1} E_{0}}{E_{0}+E_{1}} \dot{\varepsilon}
$$

where $\varepsilon$ is the induced strain, $\sigma$ is the applied stress, $\dot{\varepsilon}$ is the strain rate, $\dot{\sigma}$ is the stress rate, $E_{0}$ and $E_{l}$ are elastic modulus of the springs, and $\mu_{1}$ is viscosity of the damper element as shown in Fig. 4.a.

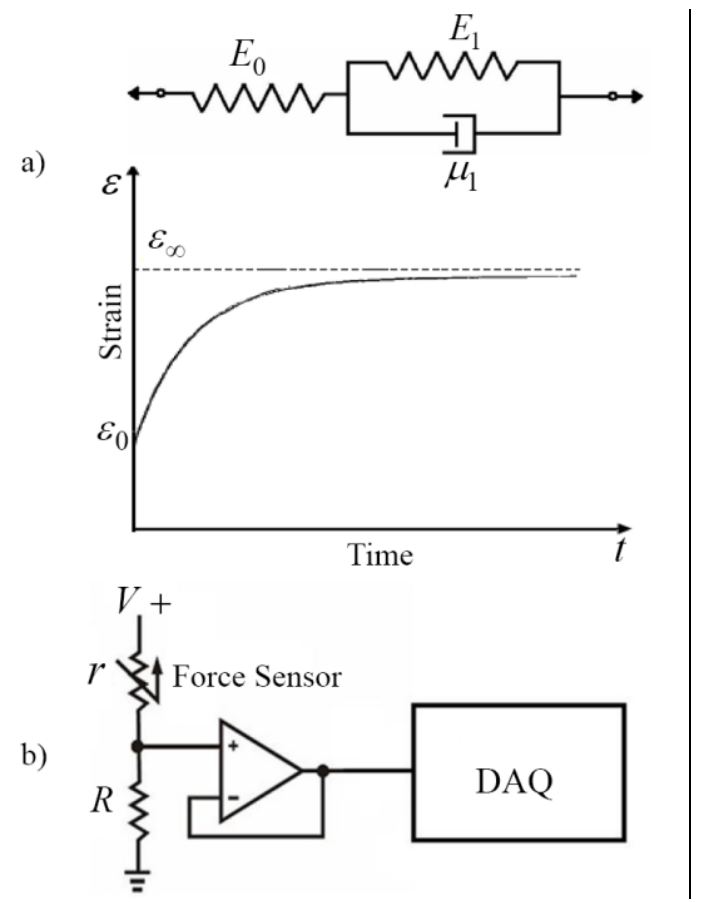

Fig. 4. a. Three parameters solid model and the creep behavior of it b. The electric circuit of a single force sensor connected

In this work only the compressive stress component is of interest which is normal to the plane of the sensor as shown in Fig. 4.a. Thus, other stress and strain components would not be taken into consideration and the main stress or force component would be in the $z$ direction.

To find $E_{0}, E_{1}, \mu_{1}$, the creep test conditions are applied to the constitutive equation (14). The equation can then be solved and the coefficients determined according to experimental data. In a creep test, a constant stress is applied to the sample and maintained for the duration of the test. Therefore, the stress is constant and the stress rate is zero. So by inserting these two conditions into (14), the constitutive equation is changed to a first order differential equation of strain. By solving the first order differential equation and by converting the stress to the corresponding force element [27], the induced strain as a function of time is written as:

$$
\varepsilon(t)=\frac{F}{A E_{0}}+\frac{F}{A E_{1}}\left(1-e^{-\frac{E_{1}}{\mu_{1}} t}\right)
$$

where $\varepsilon(t)$ is the induced strain, and $A$ is the corresponding area of the force sensor, $F$ is the applied force, $E_{0}$ and $E_{l}$ are elastic modulus of the springs, $\mu_{l}$ is viscosity of the damper element, and $t$ is the time. The stress is converted to force in (15). The coefficients $E_{0}, E_{1}, \mu_{1}$ are then determined experimentally and are calculated from the creep curve of semi-conductive polymer composites.

\section{F. Modeling of the total resistance}

By considering time dependency of strain, (15) could be substituted into (9) resulting in a new time dependent expression for the resistance of polymer. In this expression, the creep behaviour is modeled and included in the formulation as discussed before. The new formula for the resistance of polymer, including the creep effect could be written as

$$
\begin{aligned}
& R_{P o l^{*}}= \\
& R_{0}\left[1-\left(\frac{F}{A E_{0}}+\frac{F}{A E_{1}}\left(1-e^{-\frac{E_{1}}{\mu_{1}} t}\right)\right] e^{-\gamma S_{0}\left[\frac{F}{A E_{0}}+\frac{F}{A E_{1}}\left(1-e^{-\frac{E_{1}}{\mu_{1}} t}\right)\right]}\right.
\end{aligned}
$$

where $S_{0}$ can be found from (8), and $\gamma$ can be found from (4).

Therefore, the final relationship between the resistance of a semi-conductive polymer composite and the applied force taking into account the effects of contact and creep can be written as

$$
\begin{aligned}
& R_{\text {total }}=2 R_{\text {Con }}+R_{P o l^{*}}= \\
& =\frac{\rho_{1}+\rho_{2}}{2} \sqrt{\frac{\pi H}{F}}+ \\
& R_{0}\left[1-\left(\frac{F}{A E_{0}}+\frac{F}{A E_{1}}\left(1-e^{-\frac{E_{1}}{\mu_{1}} t}\right)\right] e^{-\gamma S_{0}\left[\frac{F}{A E_{0}}+\frac{F}{A E_{1}}\left(1-e^{-\frac{E_{1}}{\mu_{1}} t}\right)\right]}\right.
\end{aligned}
$$

\section{EXPERIMENTS}

\section{A. Experimental Setup}

In order to verify the validity of the proposed formulation, creep tests were performed on Linqstat from which, a force sensor was subsequently fabricated with the intention of its being used. The fabricated force sensor is intended to be used as an element of a force sensor array in minimally invasive surgery applications. The resolution of the fabricated force sensor is $0.1 \mathrm{~N}$. The formulation proposed above can be used to optimize the sensor by eliminating drift and obtaining a better output.

The fabricated force sensor, as shown in Fig. 1.a, has one input/output port. The sensor must be fed by a constant DC voltage supply. The input voltage to the sensor was set to $5 \mathrm{~V}$. The sensor was then connected to a buffer circuit which, in turn, was connected to a PC via a Data Acquisition (DAQ) board. The buffer circuit was used to reduce the loading effect 
a)

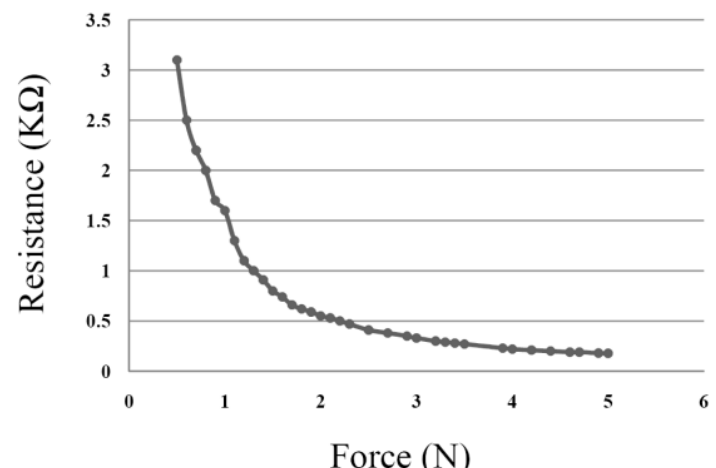

b)

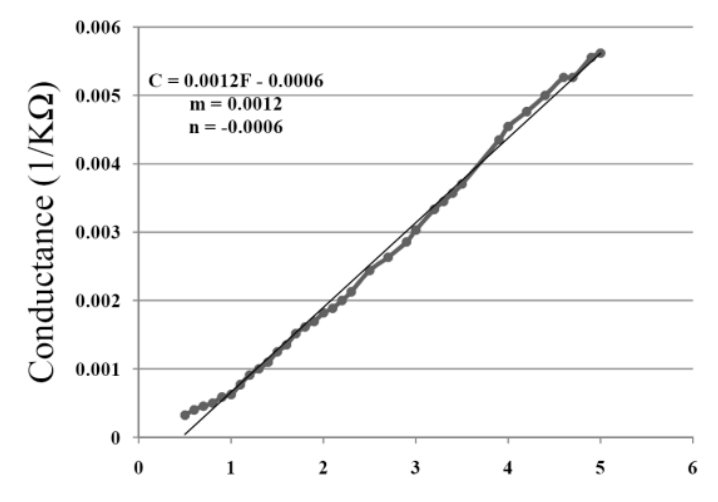

Force $(\mathrm{N})$

Fig. 5. a. Force-resistance relationship b. Force-conductance relationship

of the force sensor on the DAQ amplifiers. As a consequence, the cross talk between the different input channels of the DAQ system was minimized.

To relate the force sensor output, measuring DC voltage, to the sensed force, a calibration procedure was implemented. Experimental data showed that the force (F) applied to the sensor has a linear relationship with the conductance (C), Fig. 5.b shows the Force and Conductance relationship in which $C=m F+n$, where $m$ and $n$ are constants [32].

The circuit in Fig. 4.b was used to find the relationship between conductance and force. In order to apply accurate normal forces to the piezoresistive force sensors during calibration, an ELECTROFORCE 3200 BOSE device was used to apply a normal force to the sensor plane. As shown in Fig. 4.b, a simple formula can be obtained [32] for the inputoutput voltage relationship as:

$$
\begin{gathered}
V_{o}=\frac{R}{R+r} V^{+} \Rightarrow C=\frac{1}{r}=\frac{1}{\left(\frac{V^{+}}{V_{o}}-1\right) R} \\
\underset{\text { Substitute }}{\mathrm{C=mF+n}} F=\frac{1}{\left(\frac{V^{+}}{V_{o}}-1\right) R \cdot m}-\frac{n}{m}
\end{gathered}
$$

where $F$ is the force, $C$ is the conductance, $R$ is the resistance, and $m$ and $n$ are constants. The LabView program measures $V_{o}$ for the known force applied to the sensor. Therefore, by knowing the input voltage $V^{+}$, and the connected resistance $\mathrm{R}$, and by reading $F$ and $V_{o}$ from the Bose device and the LabView software, the two unknowns $m$ and $n$ can be found with a simple curve fitting as shown in Fig. 5. The force sensor was calibrated in the range of 0 to $5 \mathrm{~N}$ which is sufficient for the intended applications. Moreover, the creep test for the sensor is also performed in this same range. Figure 5.a shows the curve of the output of the force sensor for different applied loads. The output of the force sensor was gathered one second after application of the force at each step of the experimentation.

\section{TESTS AND DISCUSSIONS}

As mentioned earlier, several creep tests were performed on four standard samples using the ELECTROFORCE 3200 BOSE device. Figure 6.a shows the results for the creep tests. By using creep data, the viscoelastic coefficients in (15), $E_{0}$, $E_{1}$, and $\mu_{1}$, were determined for $2,4,6$, and $8 \mathrm{~N}$, respectively. Finally, three lines were fitted to each set of coefficients using the least square method. Figure 6.b shows the extracted coefficients from creep data, and fitted lines to each set of

a)

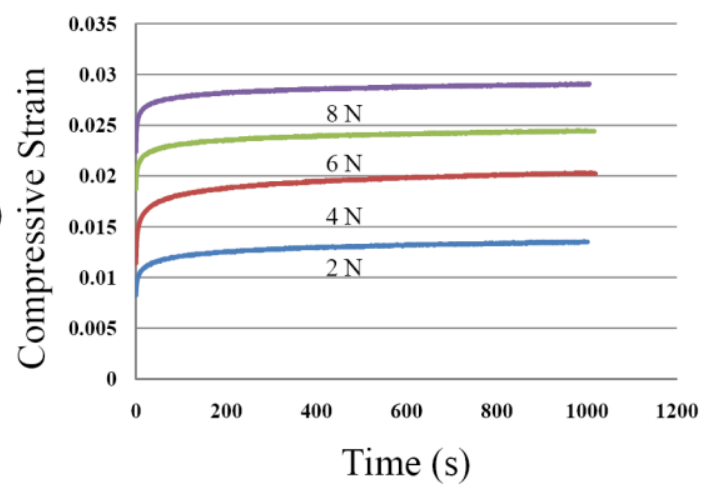

b)

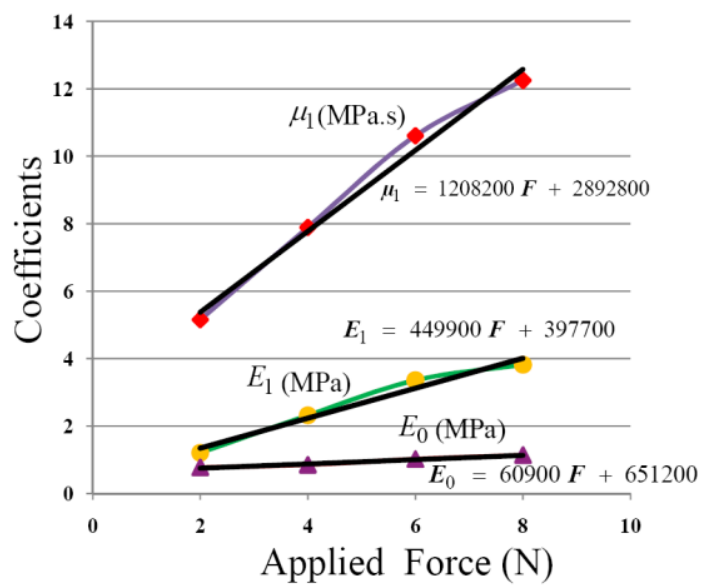

Fig. 6. a. Creep test result of Linqstat samples b. Viscoelastic coefficients for Linqstat using creep data

coefficients. By substituting the three expressions in (14), the final relation between force and resistance can be extracted for Linqstat as 


$$
\begin{aligned}
& R_{\text {total }}=\frac{\rho_{1}+\rho_{2}}{2} \sqrt{\frac{\pi H}{F}}+ \\
& R_{0}\left(\frac{F}{A}\left(\frac{e^{\left(-\frac{(449900 F+397700) t}{1208200 F+2892800}\right)}-1}{449900 F+397700}-\frac{1}{60900 F+651200}\right)+1\right) \\
& -\frac{F}{A}\left(\frac{\gamma S_{0}}{60900 F+651200}+\frac{1-e\left(-\frac{t(449900 F+397700)}{1208200 F+2892800}\right)}{449900 F+397700}\right) \\
& \times e
\end{aligned}
$$

From this formulation, the curve for the change of resistance based on applied force can be calculated for Linqstat (19). Various properties such as volume fraction and size of impregnated carbon particles, hardness of the polymer composite, and the resistivity of the polymer composites are all required parameters for this formulation. These required data were determined using different tests. Thermo Gravimetric Analysis (TGA) was performed on Linqstat samples in order to find the volume fraction of carbon particles in the polymer. A specimen was cut from a velostat sheet and was placed inside a platinum pan of the TGA device. The sample weight of Linqstat was $21.1580 \mathrm{mg}$. The test range was from $25^{\circ} \mathrm{C}$ to $1000^{\circ} \mathrm{C}$ with a $20^{\circ} \mathrm{C} / \mathrm{min}$ increase in the heating rate. In some methods of thermogravimetric analysis, nitrogen gas is introduced as a purge between the temperature range of $25^{\circ} \mathrm{C}$ to $650^{\circ} \mathrm{C}$, and then the nitrogen is switched to air from $650^{\circ} \mathrm{C}$ to $1000^{\circ} \mathrm{C}$ which results in complete oxidation of residual carbon [33]. In this case, almost no ash remains at the end. However, in the current TGA analysis, nitrogen gas was only introduced to the sample in order to preserve the residual carbon in order that it could be used later as a sample for Scanning Electron Microscopy in order to determine the size of the carbon particles. The result showed that Linqstat contained $19.89 \%$ of carbon, and $80.11 \%$ of polyethylene with additives. So, the weight of carbon particles inside the composite was equal to $4.2083 \mathrm{mg}$, and the weight of the matrix, polyethylene, was $16.9496 \mathrm{mg}$. By considering the mass density of polyethylene as an amorphous structure having a mass density of $0.910 \mathrm{gr} / \mathrm{cm}^{3}$, which is suitable for low density polyethylene, the volume of polyethylene was $0.0186 \mathrm{~cm}^{3}$. Also, by considering the mass density of carbon black as $0.56 \mathrm{gr} / \mathrm{cm}^{3}$, the volume of carbon can be calculated as being $0.0075 \mathrm{~cm}^{3}$. So, the volume fraction of the carbon inside the Linqstat is $\phi=0.2873$, which can be placed in (8).

The size of impregnated filler particles inside the nonconductive polymer is another important parameter that had to be taken into account. The size of the carbon black used in this present research was measured using Scanning Electron Microscopy (SEM). A sample of Linqstat was prepared for SEM testing by coating it with gold-palladium alloy with the thickness of $250 \mathrm{~nm}$. Then, the sample was analyzed by SEM. Figure 7 shows the carbon particles inside the sample. Carbon particles are dispersed inside the polymer like large connected colonies and it was determined that their diameter was $D=500 \mathrm{~nm}$. By having $D$ and $\phi$, the inter-particle separation between two adjacent carbon particles in Linqstat can be determined from (8).

As mentioned earlier, different devices were used to test the Linqstat and the force sensor. Figure 8 shows three of the main
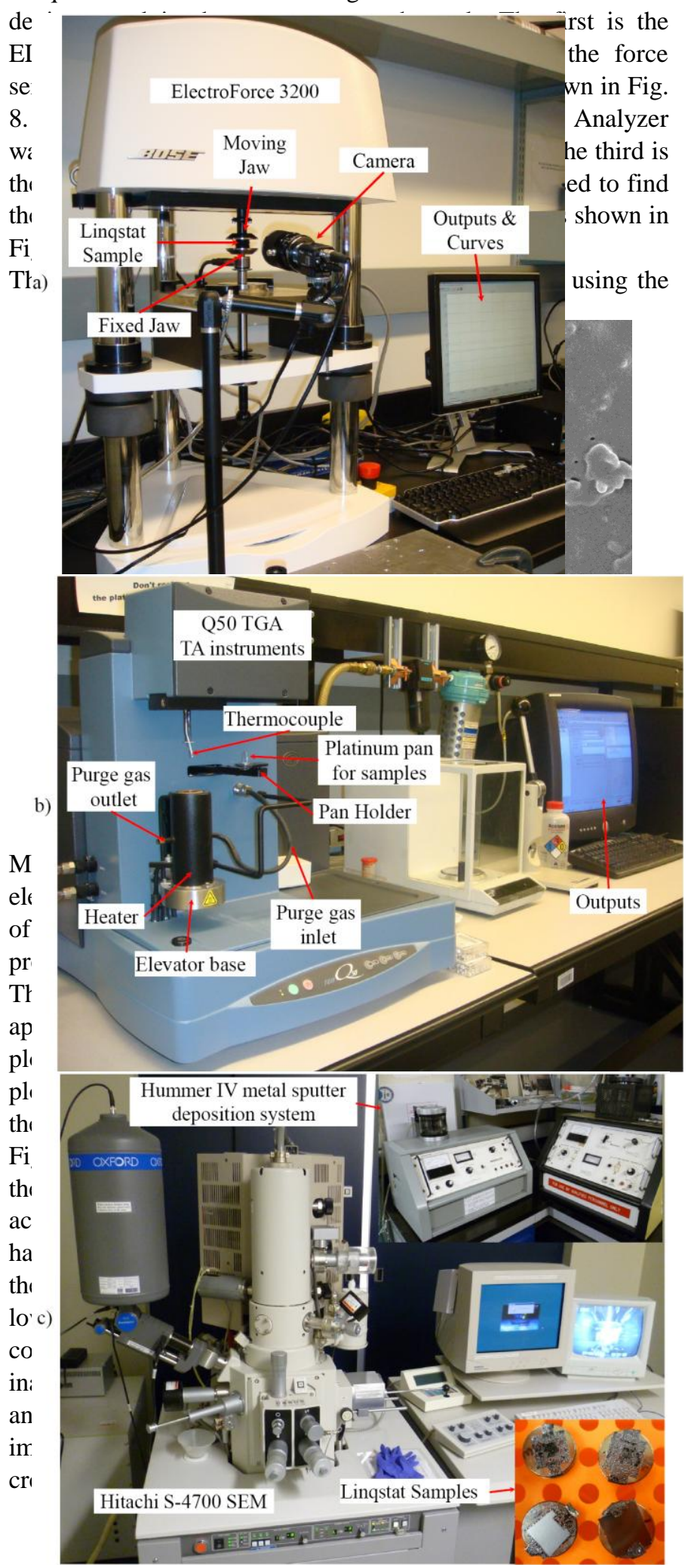

Fig. 8 .a. ELECTROFORCE 3200 BOSE device b. TA Q50

Thermogravimetric Analyzer c. Hitachi S-4700 scanning electron microscopy 
test, several constant forces with different magnitudes were applied to the force sensor. Then, the change in the resistance of the force sensor over time was recorded for each of the forces to show the creep behavior in the sensor output. The gathered data for each force were finally compared to the theoretical model prediction for that specific force as shown in Fig. 9.b. To include all the comparison results together, the chart was drawn in logarithmic scale for the resistance. As can be seen from the test results of the comparison in Fig. 9.b, the model predictions closely match the experimental results of the test.

The proposed theoretical model contains three variables namely, resistance, force, and time. The relationship of these three variables is shown in Fig. 10 as a three dimensional surface.

The piezoresistive behaviour of other kind of semi-conductive polymer composites, such as Velostat, can be predicted by the developed model. Linqstat and Velostat have some differences in the size and the volume fraction of carbon black particles, and in the type of the nonconductive polymer, resulting in different hardnesses for Velostat and Linqstat. Hence, the model can be also used for another type of semi-conductive polymer composites by populating the model with appropriate parameters.

\section{SUMMARY AND CONCLUSION}

A novel formulation has been developed for modeling the dependence of electrical resistivity of semi-conductive polymer composites on an applied load. The proposed formulation includes a viscoelastic model that shows the creep behavior within semi-conductive polymer composites. Moreover, the contact resistance was also included in the proposed formulation. Although contact resistance is not a large factor at high resistances, it turns out that it does have a significant effect in the lower range of resistance. This is very important given the fact that the working range of the sensor needs to be accurate from $0.5 \mathrm{~N}$ to $5 \mathrm{~N}$.

Linqstat as a semi-conductive polymer composite was selected to verify the validity of the proposed formulation by means of experiments. Creep tests were performed on Linqstat samples and the viscoelastic parameters were determined. In addition, by using Linqstat, an accurate force sensor was fabricated because the results obtained from the formulas corresponded very closely to the actual output readings from the sensors.

\section{REFERENCES}

[1] N. Hu, Y. Karube, C. Yan, Z. Masuda, and H. Fukunaga, "Tunneling effect in a polymer/carbon nanotube nanocomposite strain sensor", Acta Materialia, vol. 56, issue 13, pp. 2929-2936, 2008.

[2]M. Knite, V. Teteris, A. Kiploka, and J. Kaupuzs "Polyisoprene-carbon black nanocomposites as tensile strain and pressure sensor materials". Sens Actuat A Phys, vol. 110, issue 1-3, pp.142-149, 2004.

[3]S. Qu, S.C. Wong. "Piezoresistive behavior of polymer reinforced by expanded graphite". Compos Sci Technol, vol. 67, pp. 231-237, 2007.

[4]L. Wang, Ding TH, and P. Wang "Effects of compression cycles and precompression pressure on the repeatability of piezoresistivity for carbon black-filled silicone rubber composite". J Polym Sci B Polym Phys, vol. 46, pp.1050-1061, 2008.
[5]W.E. Mahmoud, A.M.Y. El-Lawindy, M.H. El Eraki, and H.H. Hassan. "Butadiene acrylonitrile rubber loaded fast extrusion furnace black as a compressive strain and pressure sensors". Sens Actuat A Phys, vol. 136, pp. 229-233, 2007.

[6]L. Wang, Ding TH, and P. Wang,"Effects of conductive phase content on critical pressure of carbon black filled silicone rubber composite". Sens Actuat A Phys, vol.135, pp. 587-592, 2007.

a)
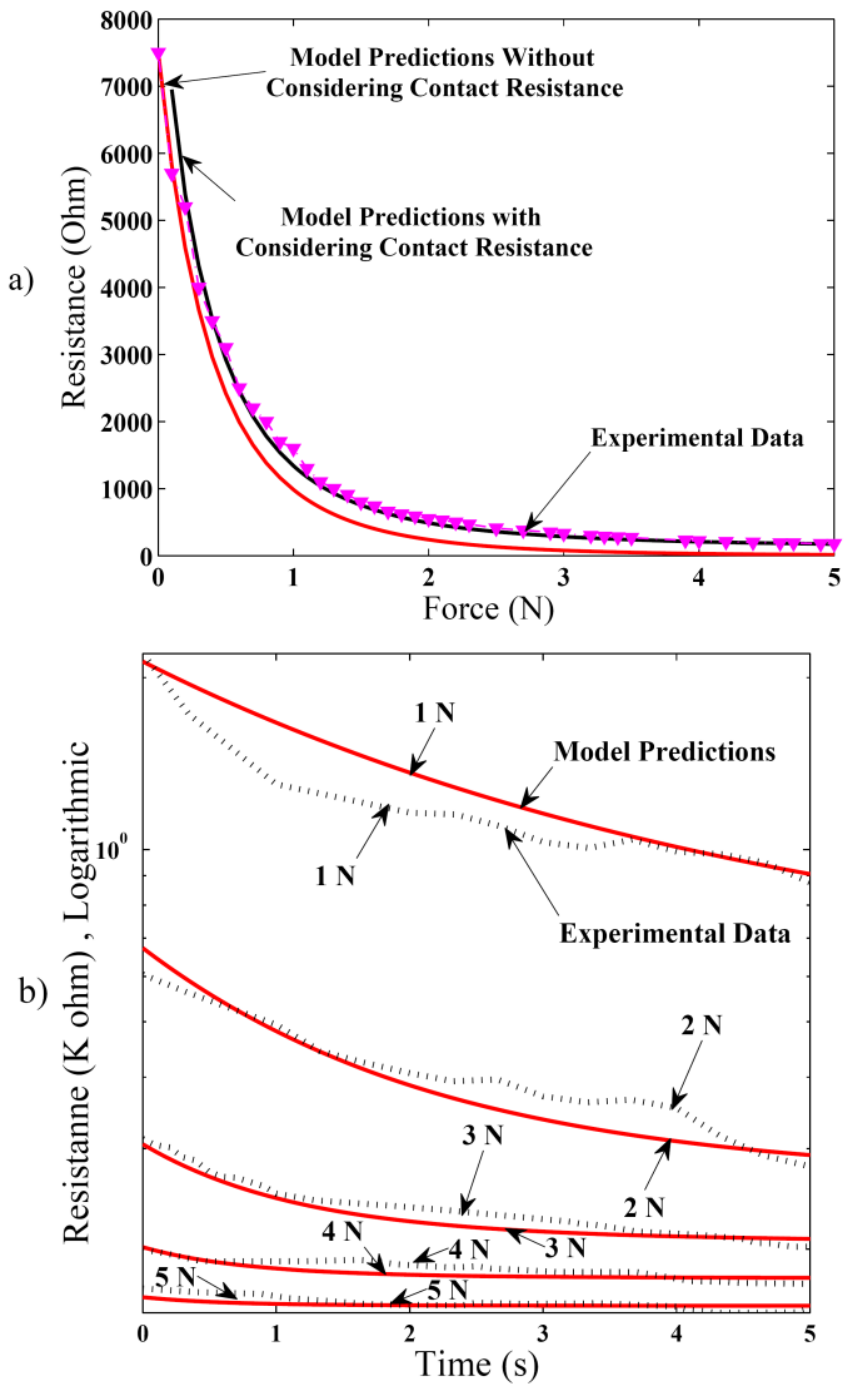

Fig. 9. a. Comparison of the experimental results and the theoretical results for change of resistance per applied force b. Comparison of the experimental results and the theoretical results for change of resistance over time for different force magnitudes 


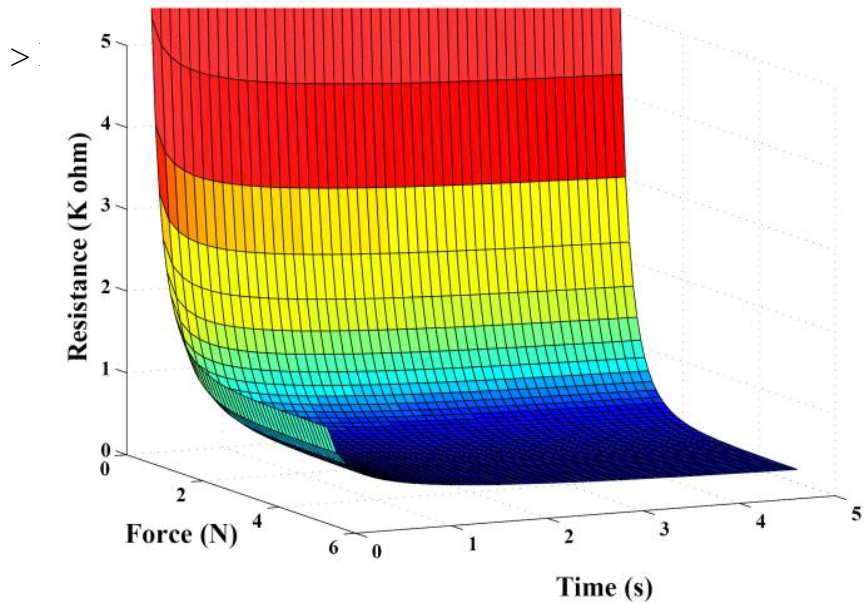

Fig. 10. Graphical representation of the proposed model

[7]C. Melchiorri, "Slip detection and control using tactile and force sensors", IEEE/ASME Transaction on Mechatronis, vol.5, pp.235-243, 2000.

[8] V. Nicola, H. Valentina, T. Mario, R. emiliano, T. Alessandro, Z. Giuseppe, G. Roger, C. Dominique, S. Nicola, S. E. Pasquale, G. Giulio, P. Vincenzo, S. M. Filomena, B. Antonio, P. Pietro, D. D. Emilio, L. Luigi, "Sensing glove for brain studies: design and assessment of its compatibility for fMRI with a robust test", IEEE/ASME Transactions on Mechatronics, vol.13, pp 345-351, 2008.

[9]H. Kawasaki, T. Komatsu, K. Uchiyama, "Dexterous anthropomorphic robot hand with distributed tactile sensor: Gifu hand II", IEEE/ASME Transactions on Mechatronics, vol.7, pp 296-303, 2002.

[10] L. R. Lin and H. P. Huang, "Integrating fuzzy control of the dexterous National Taiwan University (NTU) hand," IEEE/ASME Transactions on Mechatronics, vol. 1, pp. 216-229, Sept. 1996.

[11] M. Nilsson,"Tactile sensors and other distributed sensors with minimal wiring complexity" IEEE/ASME Trans. Mechatronics, vol.5, No.3, pp.253-257,2000.

[12] H. Z. Tan, L. A. Slivovsky, and A. Pentland, "A sensing chair using pressure distribution sensors," IEEE/ASME Transactions on Mechatronics, Vol. 6, No. 3, pp. 261-268, 2001

[13] W.J. Lee, J.H. Son, N.H. Kang, I.M. Park, and Y. H. Park, "Finiteelement analysis of deformation behaviors in random-whiskerreinforced composite", Scripta Materialia, vol. 61, pp. 580-583, 2009.

[14] G. R. Ruschau, S.Yoshikawa, and R. E. Newnham, "Resistivities of conductive composites", Journal of applied physics, vol. 72, pp. 953959, 1992

[15] L. Wang, Ding TH, and P. Wang," Influence of carbon black concentration on piezoresistivity for carbon-black-filled silicone rubber composite", Carbon, vol. 47, pp. 3151-3157, 2009.

[16] Z. Xie, Y. Young-Jin, L. Chul-Kyoung, "Simulation of electrical resistivity of carbon black filled rubber under elongation", Journal of macromolecular science, vol. 46, pp: 561-567, 2007.

[17] R. Hall, G. Desmoulin, T. Milner, "A technique for conditioning and calibrating force-sensing resistors for repeatable and reliable measurement of compressive force", Journal of Biomechanics, vol. 41, pp. 3492-3495, 2008.

[18] X. W. Zhang, Y. Pan, Z. Qiang, and Y. Xiao-Su," Time Dependence of Piezoresistance for the Conductor-Filled Polymer Composites" Journal of Polymer Science, vol. 38, pp. 2739-2749, 2000.

[19] K. Ohe, and Y. Naito "Piezoresistance Effect of Graphite-Papostor" Jpn. J. Appl. Phys.vol.10, pp. 868-872, 1971.

[20] J.G. Simmons, "Generalized Formula for the Electric Tunnel Effect between Similar Electrodes Separated by a Thin Insulating Film”, $J$. Appl. Phys,. Vol. 34, 1793.

[21] X.W. Zhang, Y. Pan, Q. Zheng, and X.S. Yi, "Piezoresistance of Conductor Filled Insulator Composites" Polymer Int., vol. 50, pp. 229 236, 2001.

[22] S. Wu," Phase structure and adhesion in polymer blends: A criterion for rubber toughening", Experimental Station, Polymer, vol. 26, pp. 1855$1863,1985$.

[23] R. Holm, Electric Contacts: Theory and Applications (Book style). Berlin: Springer, 1967.

[24] J. H. Constable, "Analysis of the Constriction Resistance in an ACF Bond," IEEE Transactions on Components and Packaging Technologies, vol. 29, pp. 494-501, 2006.
[25] R.L. Jackson, R. D. Malucci, S. Angadi and J. R. Polchow, "A simplified model of multiscale electrical contact resistance and comparison to existing closed form models", Proceedings of the $55^{\text {th }}$ IEEE Holm conference of Electrical Contacts, British Columbia, 2009, pp. 28-35.

[26] R.A. Coutu, J.R. Reid, R. Cortez, R.E. Strawser, P.E. Kladitis, "MicroSwitches with sputtered Au, AuPd, Au-on-AuPt, and AuPtCu Alloy Electric Contacts", IEEE Trans. components and packaging thechnology, vol. 29, pp. 341-349, 2006.

[27] L. Brinson, Polymer Engineering Science and Viscoelasticity: An introduction (Book style).New York: Springer, 2008.

[28] S. Govindarajan, N. A. Langrana, and G. J. Weng, "An Experimental and Theoretical Study of Creep of a Graphite/Epoxy Woven Composite", Polymer Composites, vol. 17, pp. 353-361, 1996.

[29] M. H. Abd-El Salam, "Stress-Strain and Creep Characteristics of HAF Black- Loaded NBR/EPDM Rubber Blends" Egypt. J. Solids, vol. 30, pp. $175-187,2007$

[30] A. Ranade, K. Nayak, D. Fairbrother, N. Anne, and D. Souza "Maleated and non-maleated polyethylene-montmorillonite layered silicate blown films: creep, dispersion and crystallinity" Polymer, vol. 46, pp. 73237333,2005

[31] Y. M. Wang, and G. J. Weng, "The influence of inclusion shape on the overall viscoelastic behavior of composites" ASME applied mechanics, vol. 59, pp. 510-518, 1992.

[32] M. Kalantari, M. Ramezanifard, R. Ahmadi, J. Dargahi, and J. Kövecses, "Design, Fabrication, and Testing of a Piezoresistive Hardness Sensor in Minimally Invasive Surgery" IEEE Haptics Symposium 2010, USA. to be published

[33] K. Mohamed, "Thermogravimetric Analysis, Theory, Operation, Calibration and Data Interpretation", Available: www.tainstruments.com

Masoud Kalantari completed his M.Sc. degree in Mechatronics Engineering in 2007 from Iran University of Science and Technology (IUST), Tehran, Iran. Currently, he is a $\mathrm{PhD}$ Candidate in Minimally Invasive Surgery Laboratory in Mechanical and Industrial Engineering Department at Concordia University in Montreal, Canada. He is also affiliated with the Centre for Intelligent Machines at McGill University. His research interest is Minimally Invasive Surgery, Tactile Sensing, and Haptics.

Javad Dargahi received his B.Sc., M.Sc., and Ph.D. degree in Mechanical Engineering in the UK. He was a Research Assistant at the Glasgow Caledonian University, Glasgow, UK. He was a Senior Post-Doctoral Research Associate with the Micromachining/Medical Robotics Group at Simon Fraser University, Burnaby, B.C., Canada. He is currently an Associate Professor in the Department of Mechanical and Industrial Engineering at Concordia University in Montreal, Canada.

Jozsef Kövecses is an Associate Professor of Mechanical Engineering at McGill University. $\mathrm{He}$ is also affiliated with the Centre for Intelligent Machines. His primary research interests are in the areas of Dynamics, Control, and Mechatronics. He has significant research and industrial experience in these fields, and has also published numerous papers in leading journals.

Mahmood Ghanbari Mardasi received his BS degree from the Department of Mechanical Engineering, Isfahan University of Technology, Iran in 2006 and MS degree in Mechanical Engineering, from Tarbiat Modares University, Iran in 2009. Currently, he is working toward his PhD degree at Optical BioMicrosystem laboratory, Department of Mechanical and Industrial Engineering, Concordia University, Canada. His main research interests are in the area of Micro and Nano electromechanical systems (MEMS and NEMS), Bio MEMS/NEMS and Mechatronic systems.

Shahrzad Nouri received her BS degree from the Department of Mechanical Engineering, Babol University of Technology, Iran in 2000 and MS degree in Aerospace Engineering, from Tarbiat Modares University, Iran in 2006. Currently, she is working toward her $\mathrm{PhD}$ degree at Bio-MEMS laboratory, Department of Mechanical and Industrial Engineering, Concordia University, Canada. Her main research interest is in the area of Bio-Micro and Nano electromechanical systems. 